\title{
A Visão de um Cirurgião sobre o Estudo SYNTAX
}

\author{
Fabio B. Jatene ${ }^{1}$
}

C om o progressivo desenvolvimento dos métodos intervencionistas de tratamento da doença arterial coronária, a cirurgia de revascularização do miocárdio (CRM) passou por várias fases de mudanças e adaptações. Desde o início, quando o cirurgião ficava de sobreaviso caso algum problema maior pudesse ocorrer durante a angioplastia, até os tempos atuais de disputa pelo mesmo paciente, aí incluídos os multiarteriais, observaram-se fases com maior ou menor avanços em cada um dos métodos.

A modalidade cirúrgica, método de resultados muito conhecidos e consistentes, passou a ser continuamente questionada e comparada a outras formas de tratamento e, mesmo demonstrando sua eficácia, vem sofrendo redução de seu campo de atuação. E a que isso se deve? À piora dos resultados cirúrgicos? Ao menor treinamento ou mesmo desinteresse dos cirurgiões com essa operação, com o passar dos anos? Definitivamente a resposta é não! O que ocorre é o interesse crescente em todo o mundo e em todas as áreas médicas pelos chamados métodos menos invasivos de tratamento. Mesmo nas áreas cirúrgicas, grande parte das operações é realizada pelos métodos videoscópicos, menos agressivos e de maior aceitação pelos pacientes. E há ainda o argumento, por vezes incorreto, da possibilidade de, em casos de insucesso do método menos invasivo, tentar realizar o método convencional.

O que vem fazendo a cirurgia para se adaptar aos novos tempos? Tem trabalhado de forma efetiva para reduzir a agressão cirúrgica, por meio de incisões menores, diminuição da utilização de circulação extracorpórea, evitando muitas vezes a manipulação da aorta, preocupando-se cada vez mais com durabilidade dos enxertos, extubação precoce ainda na sala de operações e menor tempo de hospitalização, entre várias outras medidas. Isso tudo, a despeito de tratar progressivamente pacientes portadores de doença cada vez mais complexa, como indivíduos mais idosos, com doenças associadas, deterioração da função ventricular ou em fases agudas da doença coronária.
Entretanto, um dos maiores problemas, na minha opinião, e que tem dificultado o direcionamento de pacientes portadores de doença coronária é a falta de padronização na indicação das condutas e a conseqüente dificuldade no encaminhamento dos pacientes para o tratamento. Vários trials têm sido divulgados, enfocando aspectos comparativos bem específicos, e as conclusões na maioria deles são bastante claras, demonstrando redução da mortalidade para cinco anos de evolução, em favor da CRM, principalmente quando há envolvimento da artéria interventricular anterior ${ }^{1}$. O New York Registry ${ }^{2}$, com dados do mundo real, e o trabalho $\mathrm{MASS}^{3}$, realizado em nosso meio, são bons exemplos disso. Entretanto, o que se observa na prática diária é que esses trabalhos e os guias de conduta que se originam a partir deles não são, em muitas situações, seguidos. É o que se convencionou chamar de indicações off-label. E isso, por vezes, ocorre com muita freqüência, em algumas situações atingindo até $70 \%$ das indicações. Se isso por um lado cria a possibilidade de individualizar o tratamento, levando em consideração fatores que não foram abordados nas pesquisas e nos trabalhos, por outro lado traz uma desorganização a essa questão. Entram em campo fatores como a experiência e a acurácia pessoais e a visão pessoal do indivíduo que vai realizar o procedimento. A opinião de muitos ou dos que se preocuparam em padronizar as condutas acaba sendo relegada e surgem os excessos e os resultados indesejáveis e negativos. Dentre os vários trials dos últimos anos, o SYNTAX ${ }^{4,5}$ (SYNergy Between PCI with TAXus and Cardiac Surgery) é mais um deles. O SYNTAX é um estudo de fase III, que foi desenhado para determinar o melhor tratamento, se intervenção coronária percutânea (ICP) ou CRM, para pacientes com doença coronária complexa. Mais de 3 mil pacientes, recrutados nos Estados Unidos e na Europa e tratados em 85 hospitais, foram analisados por uma equipe multidisciplinar, que incluía um cardiologista intervencionista e um cirurgião cardíaco, e os resultados de um ano de seguimento foram apresentados no congresso da European Society of Cardiology (ESC), na Alemanha ${ }^{6}$. Esse foi o primeiro trial a com-

\footnotetext{
1 Instituto do Coração do Hospital das Clínicas da Faculdade de Medicina da Universidade de São Paulo - São Paulo, SP. Correspondência: Fabio B. Jatene. Av. Dr. Enéas de Carvalho Aguiar, 44, 5o andar, bloco 2, sala 7 - Cerqueira César - São Paulo, SP CEP 05403-000

E-mail: fabiojatene@incor.usp.br

Recebido em: 19/9/2008 • Aceito em: 22/9/2008
} 
parar diretamente CRM e tratamento percutâneo com o uso do stent farmacológico para pacientes portadores de lesão complexa, como multiarteriais e lesão de tronco da artéria coronária esquerda. Houve dois braços principais do estudo, o da randomização e o dos registros: no primeiro, pacientes elegíveis para qualquer um dos métodos (897 para CRM e 903 para ICP), e no segundo, pacientes não elegíveis para um dos métodos (1.077 para CRM e 198 para ICP).

Esse estudo enfocou importantes aspectos e que precisam ser claramente analisados. Para se ter uma idéia de sua repercussão, o dr. Chitwood, presidente da Society of Thoracic Surgeons, considerou esse trabalho um marco para a comunidade cardiológica ${ }^{7}$.

Enfocando inicialmente o grupo randomizado, observou-se que, após um ano, os resultados da ICP foram inferiores aos da CRM, com diferença estatística em relação aos desfechos primários, aí incluídos eventos cardíacos ou cerebrovasculares adversos maiores (MACCE), como todas as causas de morte, acidente vascular cerebral (AVC), infarto e necessidade de reintervenção, que foram favoráveis ao grupo cirúrgico com 12,1\%, enquanto no grupo percutâneo foi de $17,8 \%$. Isso considerando que a evolução é de apenas um ano, de um total de cinco anos previstos, o que poderá reforçar ainda mais os dados inicialmente obtidos, como já demonstrado por Hoffman et al. ${ }^{8}$ em metanálise. Como desfechos secundários, observouse que não houve diferenças entre risco de morte ou de ataque cardíaco e houve uma diferença estatística com menor nível de AVC em favor do tratamento percutâneo ( $0,6 \%$ vs. 2,2\%). A diminuição dessa incidência de AVC só será possível quando houver maior rigor na avaliação vascular pré- e intra-operatória, principalmente das condições da porção ascendente da aorta, e que inclua análise ultra-sonográfica e medidas de fluxo cerebral intra-operatório.

O desenho de all comers do SYNTAX permitiu ainda que os pacientes considerados não elegíveis para o estudo randomizado pudessem ser incluídos em dois braços de registros, com 1.077 para CRM e 198 para ICP. Analisando agora esses registros, há também considerações interessantes a serem feitas. Nesses braços estão pacientes que foram contra-indicados para um tratamento e, conseqüentemente, submetidos a outro. Observou-se que, para MACCE, a ocorrência no registro ICP foi de $20,4 \%$, enquanto no grupo cirúrgico foi de 8,8\%. A esse respeito, o dr. Friedrich Mohr, principal investigador, de Leipzig, Alemanha, afirmou: "O tratamento percutâneo é uma opção viável para pacientes não considerados elegíveis para CRM". Por outro lado, "os resultados cirúrgicos são considerados excelentes para pacientes que não são candidatos a ICP". Além disso, outro dado evidenciado por esse estudo refere-se ao fato de a CRM ter sido a única opção de tratamento para cerca de um terço (1.077 de 3.075) de todos os pacientes considerados. Na comunidade cirúrgica, esses dados tiveram bastante repercussão e algumas são dignas de nota. O dr. Robert Guyton, chefe de Cirurgia Cardiotorácica da Emory Health Care, afirmou que os pacientes necessitam saber que, em casos como os do estudo, se, ao invés da cirurgia, o implante de stents for realizado como primeira escolha, pode levar a um índice de morte $20 \%$ a $40 \%$ maior em três anos. Disse, ainda, que os resultados desse estudo deverão alterar os padrões de utilização dos stents nos Estados Unidos, em algumas áreas em que são excessivamente empregados. Também o dr. Michael Mack, diretor médico do Baylor Heart Hospital, disse ser extremamente necessária e crítica, antes do implante de stents, a informação aos pacientes, com perfil de doença semelhante ao do estudo, sobre os riscos e benefícios, incluindo as possibilidades de diminuição da sobrevida e aumento do número de reintervenções.

Ainda em relação ao SYNTAX, há dois pontos que considero muito positivos. O primeiro refere-se à adoção do SYNTAX Score, que poderá se somar aos demais escores existentes e servir de base para uma padronização da linguagem a ser falada por todos aqueles que se dedicam a tratar desses pacientes, quer sejam clínicos, hemodinamicistas e cirurgiões, e que possa levar à uniformização das indicações e condutas atualmente tão divididas e, muitas vezes, sem padronização. O segundo ponto, inserido nesse contexto, é o conceito bastante atual de heart team, que deverá ser difundido cada vez mais e que é praticado neste trabalho, com avaliação conjunta dos casos clínicos pelos diferentes profissionais da área cardiológica. Em recente artigo, Ribeiro et al. ${ }^{9}$ comentaram sobre a importância dessa cooperação e dos evidentes benefícios para os pacientes.

Ao finalizar, entendo que o trabalho SYNTAX foi positivo, sob aspectos distintos, para as duas áreas sob análise. Para a área cirúrgica, reforçou fortemente a idéia, já externada em outros estudos, de que a CRM é um procedimento seguro, confiável e de excelentes resultados para pacientes multiarteriais e com lesão de tronco. Ao contrário do que se preconizou no passado recente de que essa operação já estaria com data marcada para seu fim, esse estudo demonstrou que sua continuidade é uma necessidade, baseada em seus resultados superiores e que realmente beneficiam os pacientes. Acho oportuna as palavras de Sir Winston Churchill ao comentar sobre a vitória britânica em El-Alamein, marco do início da derrocada das forças do Eixo na África do Norte e um dos marcos decisivos na $2^{\underline{a}}$ Guerra Mundial: “... este não é o fim, não é nem o começo do fim, mas é, talvez, o fim do começo...".

\section{REFERÊNCIAS BIBLIOGRÁFICAS}

1. Hannan EL, Wu C, Walford G, Culliford AT, Gold JP, Smith $C R$, et al. Drug-eluting stents vs. coronary-artery bypass 
grafting in multivessel coronary disease. $N$ Engl J Med. 2008;358(4):331-41.

2. Hannan EL, Racz MJ, Walford G, Jones RH, Ryan TJ, Bennett $E$, et al. Long-term outcomes of coronary-artery bypass grafting versus stent implantation. $\mathrm{N}$ Engl J Med. 2005;352(21):2174-83.

3. Hueb W, Lopes NH, Gersh BJ, Soares P, Machado LA, Jatene FB, et al. Five-year follow-up of the Medicine, Angioplasty, or Surgery Study (MASS II): a randomized controlled clinical trial of 3 therapeutic strategies for multivessel coronary artery disease. Circulation. 2007;115(9):1082-9.

4. Kappetein AP, Dawkins KD, Mohr FW, Morice MC, Mack MJ, Russell ME, et al. Current percutaneous coronary intervention and coronary artery bypass grafting practices for three-vessel and left main coronary artery disease. Insights from the SYNTAX runin phase. Eur J Cardiothorac Surg. 2006;29(4):486-91.

5. Rastan AJ, Boudriot E, Falk V, Kappetein AP, Borger MA, Serruys PW, et al. Frequency and pattern of de-novo threevessel and left main coronary artery disease; insights from single center enrolment in the SYNTAX study. Eur J Cardiothorac Surg. 2008;34(2):376-83.

6. The SYNergy between Percutaneous Coronary Intervention and Cardiac Surgery (SYNTAX) Study. In: European Society of Cardiology Congress - 2008; Munich. Anais. Munich, Germany: European Society of Cardiology Congress; 2008.

7. Society of Thoracic Surgeons. SYNTAX trial affirms bypass surgery superiority in treatment of complex coronary disease. Disponível em: http://www.sts.org/documents/pdf/ press_syntax_090208.pdf

8. Hoffman SN, TenBrook JA, Wolf MP, Pauker SG, Salem DN, Wong JB. A meta-analysis of randomized controlled trials comparing coronary artery bypass graft with percutaneous transluminal coronary angioplasty: one- to eight-year outcomes. J Am Coll Cardiol. 2003;41(8):1293-304.

9. Ribeiro EE, Dallan LAO, Ribeiro HB, Dallan LAP. Análise crítica das terapêuticas: clínica, cirurgia e cateter. Revista SOCESP. 2008;18(2):87-100. 